\title{
A 4-Week Repeated Oral Dose Toxicity Study of Ssanghwa-Tang in Crl:CD Sprague Dawley Rats
}

\author{
Sae-Rom Yoo $\mathbb{D}^{1,2}$ Hyekyung Ha ${ }^{1 D},{ }^{1}$ Mee-Young Lee, ${ }^{2}$ Hyeun-kyoo Shin ${ }^{D}{ }^{1}$ \\ Su-Cheol Han, ${ }^{3}$ and Chang-Seob Seo ${ }^{1}{ }^{1}$ \\ ${ }^{1}$ Herbal Medicine Research Division, Korea Institute of Oriental Medicine, 1672 Yuseongdae-ro, Yuseong-gu, \\ Daejeon 34054, Republic of Korea \\ ${ }^{2}$ Clinical Medicine Division, Korea Institute of Oriental Medicine, 1672 Yuseongdae-ro, Yuseong-gu, Daejeon 34054, Republic of Korea \\ ${ }^{3}$ Jeonbuk Department of Inhalation Research, Korea Institute of Toxicology, 30 Baekhak 1-gil, Jeongeup-si, \\ Jeollabuk-do 56212, Republic of Korea
}

Correspondence should be addressed to Chang-Seob Seo; csseo0914@kiom.re.kr

Received 15 February 2019; Accepted 2 May 2019; Published 16 May 2019

Academic Editor: Miguel Vilas-Boas

Copyright (C) 2019 Sae-Rom Yoo et al. This is an open access article distributed under the Creative Commons Attribution License, which permits unrestricted use, distribution, and reproduction in any medium, provided the original work is properly cited.

Ssanghwa-tang (SHT), a traditional herbal formula, has been widely used to recover fatigue or consumptive disease after an illness. Along with much attention to herbal formula, the concerns about the safety and toxicity have arisen. To establish the safety information, SHT was administrated in Crl:CD Sprague Dawley rats at a daily dose of $0,1000,2000$, and $5000 \mathrm{mg} / \mathrm{kg}$ for $4 \mathrm{weeks}$. During the test periods, we examined the mortality, clinical observation, body weight change, food consumption, organ weights, hematology, serum biochemistry, and urinalysis parameters. No changes of mortality and necropsy findings occurred in any of the groups during the experimental period. In either sex of rats treated with SHT at $5000 \mathrm{mg} / \mathrm{kg} / \mathrm{day}$, changes were observed in food intake, reticulocyte, total bilirubin, some urinalysis parameters, and relative organ weights. The results indicated that SHT did not induce toxic effects at a dose level up to $2000 \mathrm{mg} / \mathrm{kg}$ in rats. This dosage was considered no observed adverse effect level (NOAEL) and was appropriate for a 13-week subchronic toxicity study.

\section{Introduction}

Along with the increased use of herbal medicine, safety has been highlighted. Although herbal medicine has been assumed to be safe, most of the drugs may cause a side effect. To ensure the safe use of herbal medicine, there is a need to establish a safe dose range, target organ toxicity, and therapeutic window.

Ssanghwa-tang (SHT, shuanghe-tang in Chinese, Souwato in Japanese), a traditional herbal formula, has been widely used in Korea for overcoming fatigue or consumptive disease after an illness [1]. In recent studies, SHT had shown several pharmacological effects including anti-inflammatory [2], antiosteoporotic [3], and antimelanogenic activities [4].

Toxicity tests are essential for the development of new drugs and for its extended use. Acute oral toxicity test is performed early on in the development stage to determine the effect of the single dose application for 1 or 2 weeks. This procedure also determines the median lethal dose $\left(\mathrm{LD}_{50}\right)$ and provides a preliminary dose range [5]. A previous study on SHT reported its acute toxicity using Crl:CD Sprague Dawley (SD) rats and defined $\mathrm{LD}_{50}$ of SHT to be over $2000 \mathrm{mg} / \mathrm{kg} /$ day in rats, for both sexes [6].

To assess the no observed adverse effect level (NOAEL) and identify the most affected organ it is necessary to perform repeated-dose toxicity tests. These tests give information between continuous exposure of drug dose and animals, which is important for planning clinical trial [7]. To identify the relationship between the long-term exposure and the animal's response, we here assessed the 4-week repeated oral dose toxicity of SHT in SD rats. In this study, we investigated toxicity at higher dose $(5000 \mathrm{mg} / \mathrm{kg} /$ day $)$ than the one uses by Kim et al's study. This study aimed to build toxicity information, including NOAEL, and select a proper dose for subchronic toxicity test. 


\section{Materials and Methods}

2.1. Preparations of SHT Water Extract. In order to obtain the powdered SHT decoction, the 9 raw materials, Paeoniae Radix (37.50 kg), Rehmanniae Radix Preparata (15.00 kg), Astragali Radix (15.00 kg), Angelicae Gigantis Radix (15.00 $\mathrm{kg}$ ), Cnidii Rhizoma (15.00 kg), Zingiberis Rhizoma Recens $(15.00 \mathrm{~kg})$, Zizyphi Fructus $(15.00 \mathrm{~kg})$, Cinnamomi Cortex $(11.25 \mathrm{~kg})$, and Glycyrrhizae Radix et Rhizoma (11.25 kg), were purchased from Kwangmyungdag Medicinal Herbs (Ulsan, Korea), mixed, and extracted with 1,500 L of distilled water at $80^{\circ} \mathrm{C}$ for $2 \mathrm{~h}$ using reflux method. This preparation was conducted in Sungil Bioex Co. Ltd. (Hwaseong, Korea). The extracted water solution was spray-dried to make a powdered sample (39.0 kg, yield: $26.0 \%)$.

2.2. High-Performance Liquid Chromatography (HPLC) Analysis of SHT. Albiflorin (PubChem CID: 51346141, purity 99.8\%), paeoniflorin (PubChem CID: 442534, purity 98.8\%), ferulic acid (PubChem CID: 445858, purity 98.0\%), liquiritin (PubChem CID: 503737, purity 99.6\%), and glycyrrhizin (PubChem CID: 14982, purity 99.0\%) were purchased from Wako Pure Chemical (Osaka, Japan). Cinnamic acid (PubChem CID: 444539, purity 99.0\%), coumarin (PubChem CID: 323, purity 99.0\%), and 5-(hydroxymethyl)-2furaldehyde (5-HMF, PubChem CID: 237332, purity 99.0\%) were purchased from Merck KGaA (Darmstadt, Germany). Nodakenin (PubChem CID: 73191, purity 99.5\%) and liquiritin apioside (PubChem CID: 10076238, purity 98.0\%) were purchased from ChemFaces Biochemical (Wuhan, China) and Shanghai Sunny Biotech (Shanghai, China), respectively. HPLC-grade methanol, acetonitrile, and water were purchased from J.T. Baker (Phillipsburg, NJ, USA). Glacial acetic acid for HPLC was analytical grade reagent, purchased from Merck KGaA (Darmstadt, Germany). The standard solution of each reference standard was prepared at a concentration of $1000.0 \mu \mathrm{g} / \mathrm{mL}$ using methanol and was stored in the refrigerator. The HPLC analysis of extracted SHT sample was performed by modifying the previous study [8]. Briefly, HPLC system used was a Shimadzu Prominence LC-20A series (Kyoto, Japan) coupled with photo-diode array (PDA) detector. The ten marker components were separated using Gemini $\mathrm{C}_{18}(250 \mathrm{~mm} \times 4.6 \mathrm{~mm} ; 5 \mu \mathrm{m}$, Phenomenex, Torrance, CA, USA) column maintained at $40^{\circ} \mathrm{C}$. Mobile phases composed of distilled water (A) and acetonitrile (B), both with $1.0 \%(\mathrm{v} / \mathrm{v})$ acetic acid, were flowed as follows: $5-60 \%$ B for $0-40 \mathrm{~min}, 60-100 \%$ B for $40-45 \mathrm{~min}, 100 \%$ B for $45-50 \mathrm{~min}$, and $100-5 \% \mathrm{~B}$ for $50-55 \mathrm{~min}$. The analysis was carried out at a flow rate of $1.0 \mathrm{~mL} / \mathrm{min}$ and an injection volume of $10 \mu \mathrm{L}$.

2.3. Animals and Maintenance. The animal maintenance was performed in the Korea Institute of Toxicology (earned AAALAC International accreditation in 2014). This study was conducted under the approval of the Institutional Animal Care and Use Committee (approval number: G217011) according to the "Guidelines for Toxicity Tests for Drugs and Related Materials, Document \#2015-82" as prepared by the Korean Ministry of Food and Drug Safety.
Five-week-old Crl:CD SD rats ( $\mathrm{n}=20 /$ each of 10 female and 10 male) were obtained from Orient Bio Co. (Orient Bio Co., Ltd., Seongnam, Republic of Korea). All animals were housed under the controlled conditions of temperature $\left(23 \pm 3^{\circ} \mathrm{C}\right)$ and relative humidity $(50 \pm 10 \%)$ with a $12-\mathrm{h}$ light/dark cycle, a light intensity of 150-300 Lux, and 10-20 air changes per hour. The animals were kept in stainlesssteel cage for the observation period and allowed rodent diet (Catalog No. 5053, PMI Nutrition International LLC., MO, USA) and sterilized tap water ad libitum.

2.4. Group Assignment and Experimental Treatment. In a previous single oral dose toxicity test, SHT did not induce any toxic effect at dose up to $2000 \mathrm{mg} / \mathrm{kg}$ in Crl:CD rats [6] or $5000 \mathrm{mg} / \mathrm{kg}$ in CD-1 mice [9]. Considering the solubility of SHT and previous results, the maximum feasible dose was chosen at $5000 \mathrm{mg} / \mathrm{kg} /$ day. Based on the last body weight, healthy animals were randomly divided into four groups (n=5/group): SHT 1000, 2000, and $5000 \mathrm{mg} / \mathrm{kg} /$ day groups and a vehicle control group.

SHT was suspended in distilled water prior to each administration. The daily administration volume $(10 \mathrm{~mL} / \mathrm{kg})$ was calculated based on the recent body weight of the individual animal. The vehicle control group had received distilled water.

2.5. General Observation. Mortality and clinical signs were monitored twice a day (before and after treatment) throughout the experimental period. The body weight and food intake were recorded once a week during the study period. Daily food consumption was calculated as the weight of the supplied diet and remaining in cages.

2.6. Clinical Pathology and Necropsy. Clinical pathology and necropsy method have been provided in a previous report [10]. Urine samples were collected at approximately $17 \mathrm{~h}$ in metabolic cages, and analyzed using Cobas U411 urine analyzer (Roche, Berlin, Germany), Combur 10 TM urine sticks (Roche), and TBA 120FR automated chemistry analyzer (Toshiba Co., Tokyo, Japan).

Animals were fasted over $17 \mathrm{~h}$ period prior to blood collection. Blood was taken from the postcaval vein under isoflurane anesthesia. Some of the blood samples were collected in a tube coated EDTA-2K. And then, we analyzed leukocyte count (WBC), total red blood cell (RBC), hemoglobin (HGB), hematocrit (HCT), mean corpuscular volume $(\mathrm{MCV})$, mean corpuscular hemoglobin $(\mathrm{MCH})$, mean corpuscular hemoglobin concentration (MCHC), relative reticulocyte (RET\%), platelet count (PLT), and large unstained cells (LUC) using ADVIA2120i hematology analyzer (Siemens Healthcare, Erlangen, Germany). The rest of the blood samples were collected in a tube containing $3.2 \%$ sodium citrate. And then, we measured prothrombin time (PT) and activated partial thromboplastin time (APTT) using ACL Elite Pro coagulation analyzer (Instrumentation Laboratory, Milan, Italy).

For serum clinical chemistry parameters, collected blood samples were centrifuged at $3000 \mathrm{rpm}$ for $10 \mathrm{~min}$. And 
TABLE 1: Stability of ten marker compounds for 10 days in the SHT decoction $(n=3)$.

\begin{tabular}{|c|c|c|c|c|c|}
\hline \multirow{2}{*}{ Compound } & \multicolumn{5}{|c|}{ Day (\%) } \\
\hline & 0 & 1 & 4 & 7 & 10 \\
\hline 5-HMF & 100.0 & 102.2 & 104.0 & 101.4 & 98.9 \\
\hline Albiflorin & 100.0 & 103.6 & 104.3 & 101.0 & 99.9 \\
\hline Paeoniflorin & 100.0 & 98.1 & 99.7 & 96.1 & 95.9 \\
\hline Liquiritin apioside & 100.0 & 100.3 & 99.7 & 101.1 & 98.1 \\
\hline Liquiritin & 100.0 & 103.8 & 96.2 & 101.3 & 96.3 \\
\hline Ferulic acid & 100.0 & 99.7 & 103.0 & 101.6 & 99.5 \\
\hline Nodakenin & 100.0 & 100.7 & 100.4 & 101.4 & 100.2 \\
\hline Coumarin & 100.0 & 99.3 & 97.9 & 101.5 & 99.0 \\
\hline Cinnamic acid & 100.0 & 102.1 & 102.2 & 104.0 & 103.8 \\
\hline Glycyrrhizin & 100.0 & 100.7 & 100.5 & 99.7 & 105.5 \\
\hline
\end{tabular}

then, we analyzed blood urea nitrogen (BUN), creatine kinase (CK), albumin/globulin ratio (AG), aspartate aminotransferase (AST), alanine aminotransferase (ALT), gammaglutamyl transpeptidase (GGT), total bilirubin (TB), total cholesterol (TCHO), triglyceride (TG), phospholipid (PL), and alkaline phosphatase (ALP) using a Toshiba 120 FR chemistry analyzer (Toshiba Co.).

After blood collection, the animals were euthanized by exsanguination from the postcaval vein and aorta. Absolute organs were measured and relative organs weights were calculated as a ratio of the following organs: brain, heart, lung, kidneys, liver, spleen, reproductive organs, thymus, thyroid and parathyroid glands, pituitary gland, adrenal gland, seminal vesicles with coagulating gland, and salivary glands.

2.7. Statistical Analysis. Data are expressed as mean \pm standard deviation. The Bartlett's test was used to test the homogeneity of variances. When data had equal variance, group differences were assessed by a one-way analysis of variance (ANOVA) and a post hoc Dunnett's test using Pristima System (Version 7.2, Xybion, NJ, USA). When data variances were not equal, group differences were assessed by the Kruskal-Wallis test and a post hoc Dunn rank sum test.

\section{Results}

3.1. HPLC Analysis of SHT Decoction. The established HPLCPDA method was successfully applied to the quantitative analysis of the ten marker components in SHT. The stability of ten marker components in the extracted SHT was measured for 10 days (at $0,1,4,7$, and 10 days, respectively) using the prepared sample solution. As a result, the stability of all components was maintained at $95.9-105.5 \%$ as compared with the initial content at day 0 (Table 1). Ten marker components were eluted within $40 \mathrm{~min}$ and retention times of these components, 5-HMF, albiflorin, paeoniflorin, liquiritin apioside, liquiritin, ferulic acid, nodakenin, coumarin, cinnamic acid, and glycyrrhizin, were $7.85,15.70,16.62,18.34$, $18.76,19.32$, 19.76, 24.24, 27.74, and $38.50 \mathrm{~min}$, respectively (Figure 1). The amounts of ten marker components in SHT decoction at 0,1 , and 4 weeks were in the range of $0.09-15.68$, 0.09-15.63, and 0.09-15.81 mg/extract g (Table 2).
3.2. Mortality and Clinical Signs. No changes of mortality occurred in any of the groups during the experimental period (Table 3). Salivation was observed in male rats treated with SHT at $5000 \mathrm{mg} / \mathrm{kg} /$ day after $18-19$ or 24 days treatment (Table 3).

3.3. Changes in Body Weight and Food Intake. In male rats, there was no difference in body weight changes upon treatment with SHT (Figure 2(a)). But, in female rats, the body weight tended to decrease at treatment at a dose of 5000 $\mathrm{mg} / \mathrm{kg} /$ day of SHT after 22 days (Figure 2(b)).

Food intake tended to decrease in male rats treated with SHT at a dose of $5000 \mathrm{mg} / \mathrm{kg} /$ day following 12 days of treatment (Figure 3(a)). In female rats, significant differences were observed following treatment with $5000 \mathrm{mg} / \mathrm{kg} /$ day (days 12, 19) and $1000 \mathrm{mg} / \mathrm{kg} /$ day (day 19) of SHT (Figure 3(b)).

3.4. Clinical Pathology. The level of RET\% was increased in female rats treated with $5000 \mathrm{mg} / \mathrm{kg} /$ day of SHT (Table 4). The level of TB was increased in male and female rats treated with $5000 \mathrm{mg} / \mathrm{kg} /$ day of SHT. The level of ALT was decreased in male rats treated with $1000 \mathrm{mg} / \mathrm{kg} /$ day of SHT (Table 5). In urinalysis, the level of urine sodium was decreased in male rats treated with $5000 \mathrm{mg} / \mathrm{kg} /$ day of SHT. Urine bilirubin was observed in a male or female rat with $2000 \mathrm{mg} / \mathrm{kg} /$ day group, and in four male or five female rats in $5000 \mathrm{mg} / \mathrm{kg} / \mathrm{day}$ group. Urobilinogen was observed in female rats in $2000 \mathrm{mg} / \mathrm{kg} / \mathrm{day}$ group $(\mathrm{n}=1)$ and in $5000 \mathrm{mg} / \mathrm{kg} /$ day group $(\mathrm{n}=3)$ (Table 6).

3.5. Necropsy Findings. No treatment-related gross finding was observed in any rat. Relative liver weights were increased in rats of both sexes treated with $5000 \mathrm{mg} / \mathrm{kg} /$ day of SHT (Table 7). In female rats, relative weights of kidneys and spleen were increased in the group treated with 5000 $\mathrm{mg} / \mathrm{kg} /$ day of SHT (Table 7).

\section{Discussion}

Herbal formulas, a complex mixture of herbs, have been widely used in the traditional clinic. They are prescribed based on the individual's constitution and taken for various days depending on symptoms. However, many of herbal 


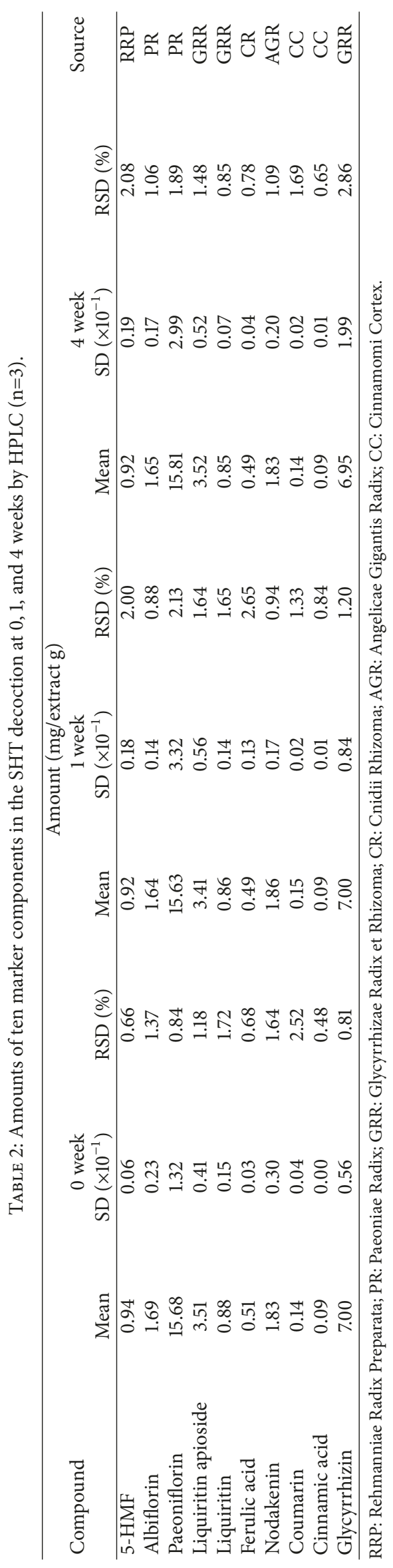


TABLE 3: Mortality and clinical signs in rats treated with SHT for 4 weeks.

\begin{tabular}{|c|c|c|c|c|c|c|}
\hline \multirow{2}{*}{ Group } & \multicolumn{5}{|c|}{ Dosing phase } & \multirow{2}{*}{ Salivation $^{\mathrm{b}}$} \\
\hline & 1 day & $\leq 1$ weeks & $\leq 2$ weeks & $\leq 4$ weeks & Final mortality ${ }^{\mathrm{a}}$ & \\
\hline \multicolumn{7}{|l|}{ Male } \\
\hline $0 \mathrm{mg} / \mathrm{kg} /$ day & 0 & 0 & 0 & 0 & $0 / 5$ & $0 / 5$ \\
\hline $1000 \mathrm{mg} / \mathrm{kg} /$ day & 0 & 0 & 0 & 0 & $0 / 5$ & $0 / 5$ \\
\hline $2000 \mathrm{mg} / \mathrm{kg} /$ day & 0 & 0 & 0 & 0 & $0 / 5$ & $0 / 5$ \\
\hline $5000 \mathrm{mg} / \mathrm{kg} /$ day & 0 & 0 & 0 & 0 & $0 / 5$ & $5 / 5$ \\
\hline \multicolumn{7}{|l|}{ Female } \\
\hline $0 \mathrm{mg} / \mathrm{kg} /$ day & 0 & 0 & 0 & 0 & $0 / 5$ & $0 / 5$ \\
\hline $1000 \mathrm{mg} / \mathrm{kg} /$ day & 0 & 0 & 0 & 0 & $0 / 5$ & $0 / 5$ \\
\hline $2000 \mathrm{mg} / \mathrm{kg} /$ day & 0 & 0 & 0 & 0 & $0 / 5$ & $0 / 5$ \\
\hline $5000 \mathrm{mg} / \mathrm{kg} /$ day & 0 & 0 & 0 & 0 & $0 / 5$ & $0 / 5$ \\
\hline
\end{tabular}

${ }^{a}$ Number of animals with dead animals/total animal number.

${ }^{\mathrm{b}}$ Number of animals with sign animals/total animal number.

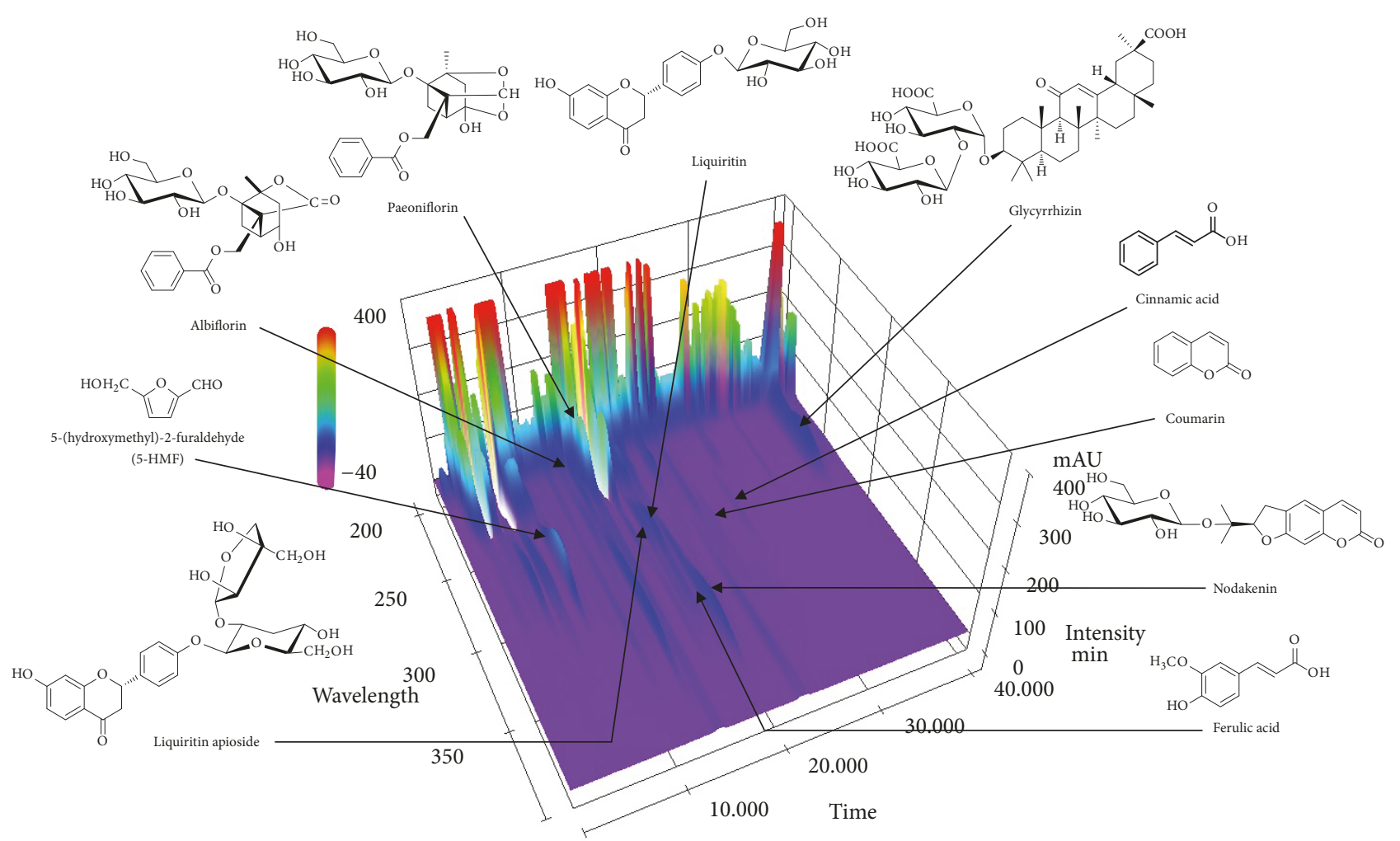

FIGURE 1: Three-dimensional HPLC chromatogram of the SHT samples using HPLC-PDA.

formulas have not been subjected to toxicity tests. Drug toxicity is closely related to dosage and duration of exposure. Previous studies reported that SHT had no toxic effect at a dose up to $2000 \mathrm{mg} / \mathrm{kg}$ in Crl:CD rats [6] or $5000 \mathrm{mg} / \mathrm{kg}$ in CD-1 mice [9] in acute toxicity tests. In this study, we administrated SHT for 4 weeks in Crl:CD SD rats to evaluate subacute toxicity. The SHT treatment did not induce adverse changes in mortality, the body weight of male rats, and gross findings. Minor changes were observed in some of the rats treated with SHT.
The bitter taste is the most common flavor in the herbal formula, which induces secretion of saliva. Although salivation was observed in male rats treated with $5000 \mathrm{mg} / \mathrm{kg} /$ day of SHT, it was not accompanied with significant changes in salivary gland between the control group and the SHT-treated group at a dose of $5000 \mathrm{mg} / \mathrm{kg} /$ day. Therefore, we regarded the salivation, by administration of SHT, to be caused only by its bitter taste.

Oral administration of $5000 \mathrm{mg} / \mathrm{kg} /$ day of SHT significantly decreased the food intake in female rats at 15 and 


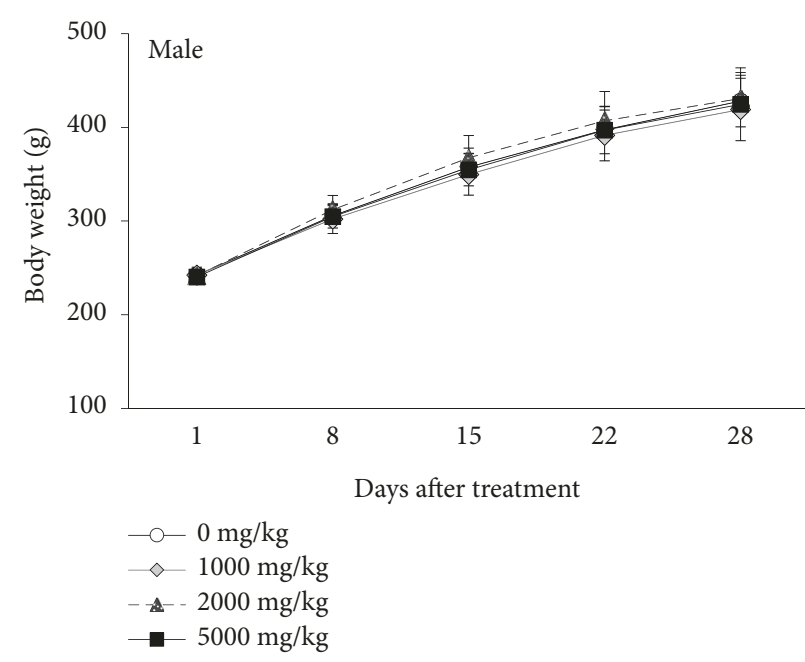

(a)

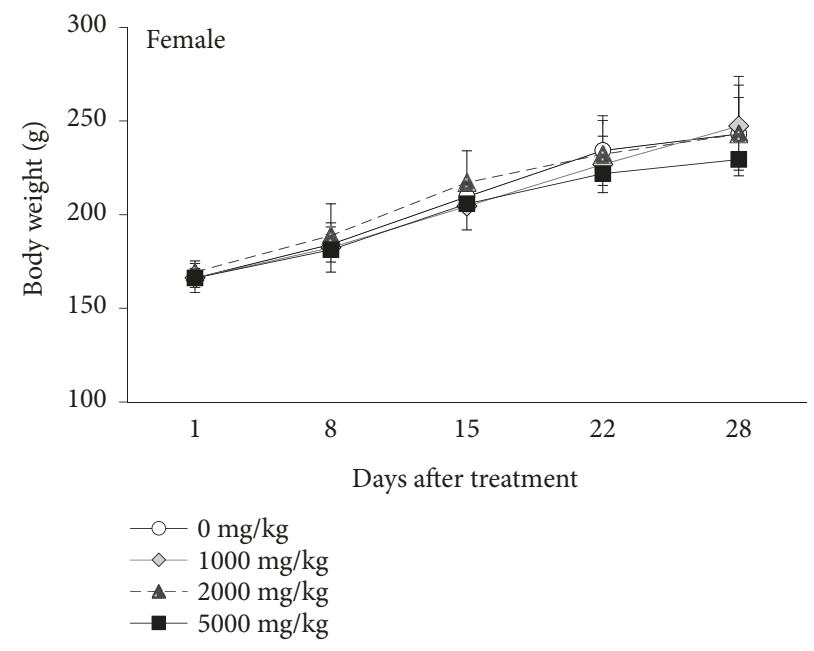

(b)

FIGURE 2: Body weight changes of male (a) and female (b) Crl:CD SD rats administered with the SHT at dose level of 0, 50, 100, and 2000 $\mathrm{mg} / \mathrm{kg} /$ day for 4 weeks. Results are presented as mean $\pm \mathrm{SD}$.

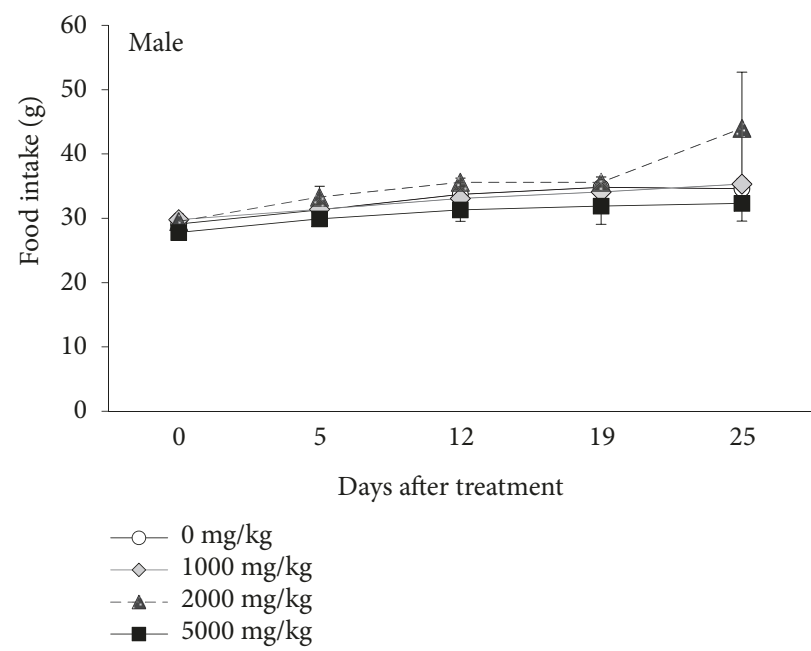

(a)

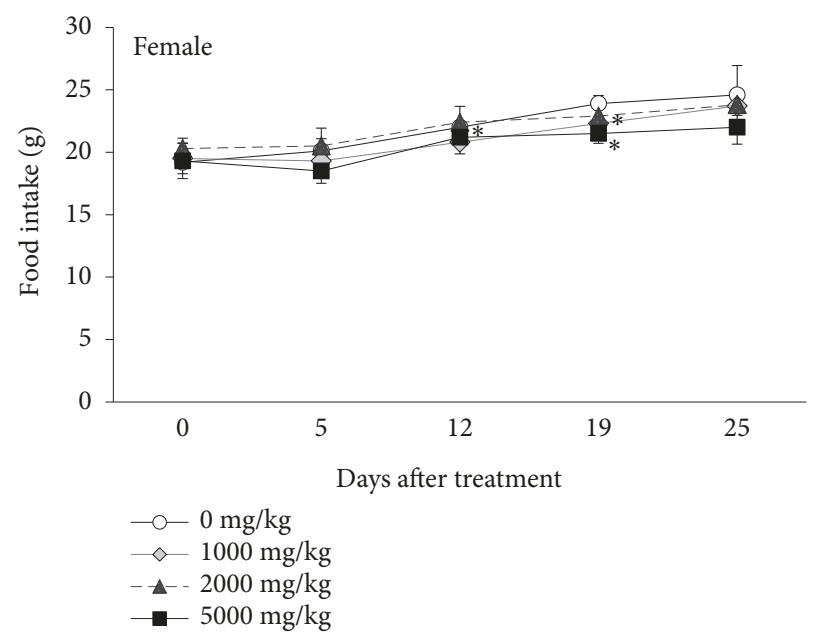

(b)

FIGURE 3: Food intakes of male (a) and female (b) Crl:CD SD rats administered with the SHT at dose level of 0, 50, 100, and 2000 mg/kg/day for 4 weeks. Results are presented as mean \pm SD. $*$ Means significant at the $p<0.05$ level.

22 days, which might also decrease the body weight. This finding did not show a dose-dependent correlation. Thus, any alteration in food intake and body weight in treated groups was regarded as incidental and not a sign of toxicity.

In $5000 \mathrm{mg} / \mathrm{kg} /$ day group, increased level of RET\% in female rats, increased level of TB, and detection of urine bilirubin and urobilinogen might be considered as the SHTmediated changes. A reticulocyte count evaluates bone marrow ability in erythropoiesis along with other tests including RBCs, HBG, and HCT. Although administration of SHT at a dose of $5000 \mathrm{mg} / \mathrm{kg} /$ day in female rats increased the RET\%, it did not affect RBC level, nor the levels of HGB and HCT [11]. The normal or expected ranges for TB levels are as follows:
0.10-1.00 mg/dL for males and $0.20-1.00 \mathrm{mg} / \mathrm{dL}$ for females [12]. Although TB levels were significantly increased at the highest dose of SHT group compared to the control group, changes in TB level were within the normal ranges. Increased relative liver weight was observed in both sexes of rats treated with SHT at $5000 \mathrm{mg} / \mathrm{kg} /$ day; however, no changes were observed in gross findings and serum biochemical parameters of liver function such as AST, ALT, and GGT.

Measurement of the urinary electrolytes evaluates the renal function. Low urine sodium concentration was observed in the male rat treated with SHT at a dose of $5000 \mathrm{mg} / \mathrm{kg} /$ day. However, SHT did not change the serum electrolytes. Changes in kidney and spleen weight due to SHT 
TABLE 4: Hematological parameters of rats treated with SHT for 4 weeks.

\begin{tabular}{|c|c|c|c|c|}
\hline Dose (mg/kg/day) & 0 & 1000 & 2000 & 5000 \\
\hline \multicolumn{5}{|l|}{ Male } \\
\hline WBC $\left(10^{3} / \mu \mathrm{L}\right)$ & $9.63 \pm 1.77$ & $7.83 \pm 1.14$ & $8.94 \pm 1.13$ & $9.56 \pm 2.18$ \\
\hline $\mathrm{RBC}\left(10^{6} / \mu \mathrm{L}\right)$ & $8.09 \pm 0.26$ & $7.88 \pm 0.22$ & $8.15 \pm 0.20$ & $7.82 \pm 0.26$ \\
\hline HGB (g/dL) & $15.7 \pm 0.47$ & $15.5 \pm 0.43$ & $15.8 \pm 0.64$ & $15.3 \pm 0.29$ \\
\hline HCT (\%) & $47.9 \pm 1.87$ & $47.1 \pm 1.34$ & $48.5 \pm 1.98$ & $46.6 \pm 0.66$ \\
\hline MCV (fL) & $59.3 \pm 0.90$ & $59.8 \pm 1.66$ & $59.5 \pm 1.81$ & $59.6 \pm 1.50$ \\
\hline $\mathrm{MCH}(\mathrm{pg})$ & $19.4 \pm 0.26$ & $19.6 \pm 0.53$ & $19.4 \pm 0.52$ & $19.5 \pm 0.67$ \\
\hline $\mathrm{MCHC}(\mathrm{g} / \mathrm{dL})$ & $32.7 \pm 0.44$ & $32.8 \pm 0.08$ & $32.6 \pm 0.53$ & $32.8 \pm 0.46$ \\
\hline RET\% (\%) & $2.52 \pm 0.35$ & $2.53 \pm 0.19$ & $2.37 \pm 0.42$ & $3.38 \pm 0.95$ \\
\hline $\operatorname{PLT}\left(10^{3} / \mu \mathrm{L}\right)$ & $1013.4 \pm 53.30$ & $1047.4 \pm 88.88$ & $908.0 \pm 58.12$ & $1027.8 \pm 96.13$ \\
\hline $\mathrm{PT}(\mathrm{sec})$ & $14.8 \pm 0.54$ & $14.5 \pm 0.45$ & $15.2 \pm 0.76$ & $14.6 \pm 0.61$ \\
\hline APTT (sec) & $16.1 \pm 0.23$ & $16.0 \pm 0.92$ & $16.3 \pm 0.94$ & $15.4 \pm 0.47$ \\
\hline Neutrophils (\%) & $13.3 \pm 3.41$ & $13.1 \pm 3.55$ & $12.2 \pm 2.94$ & $12.1 \pm 4.87$ \\
\hline Lymphocytes (\%) & $81.9 \pm 4.63$ & $82.4 \pm 4.29$ & $82.8 \pm 3.70$ & $82.9 \pm 5.71$ \\
\hline Eosinophils (\%) & $1.0 \pm 0.23$ & $1.0 \pm 0.19$ & $1.2 \pm 0.50$ & $0.9 \pm 0.39$ \\
\hline Monocytes (\%) & $2.3 \pm 1.11$ & $1.9 \pm 0.41$ & $2.2 \pm 0.59$ & $2.2 \pm 0.70$ \\
\hline Basophils (\%) & $0.3 \pm 0.10$ & $0.5 \pm 0.29$ & $0.3 \pm 0.13$ & $0.3 \pm 0.05$ \\
\hline LUC (\%) & $1.0 \pm 0.18$ & $1.2 \pm 0.55$ & $1.2 \pm 0.19$ & $1.6 \pm 0.67$ \\
\hline \multicolumn{5}{|l|}{ Female } \\
\hline $\mathrm{WBC}\left(10^{3} / \mu \mathrm{L}\right)$ & $6.77 \pm 1.121$ & $7.00 \pm 1.406$ & $5.69 \pm 1.119$ & $6.46 \pm 1.433$ \\
\hline $\mathrm{RBC}\left(10^{6} / \mu \mathrm{L}\right)$ & $8.16 \pm 0.498$ & $8.17 \pm 0.460$ & $8.22 \pm 0.335$ & $7.62 \pm 0.370$ \\
\hline HGB (g/dL) & $15.8 \pm 0.52$ & $15.9 \pm 0.67$ & $15.9 \pm 0.58$ & $14.8 \pm 0.71$ \\
\hline HCT (\%) & $48.2 \pm 2.24$ & $48.0 \pm 1.59$ & $48.5 \pm 1.46$ & $45.6 \pm 2.02$ \\
\hline MCV (fL) & $59.1 \pm 1.42$ & $58.8 \pm 1.75$ & $59.1 \pm 0.83$ & $59.9 \pm 1.89$ \\
\hline $\mathrm{MCH}(\mathrm{pg})$ & $19.5 \pm 0.72$ & $19.5 \pm 0.61$ & $19.4 \pm 0.45$ & $19.5 \pm 0.76$ \\
\hline $\mathrm{MCHC}(\mathrm{g} / \mathrm{dL})$ & $32.9 \pm 0.96$ & $33.2 \pm 0.51$ & $32.8 \pm 0.67$ & $32.5 \pm 0.58$ \\
\hline RET\% (\%) & $2.10 \pm 0.20$ & $2.50 \pm 0.85$ & $2.71 \pm 0.78$ & $4.76^{*} \pm 1.40$ \\
\hline $\operatorname{PLT}\left(10^{3} / \mu \mathrm{L}\right)$ & $1006.6 \pm 125.33$ & $1106.4 \pm 102.83$ & $991.2 \pm 160.96$ & $1012.2 \pm 117.65$ \\
\hline $\mathrm{PT}(\mathrm{sec})$ & $14.7 \pm 0.54$ & $15.2 \pm 0.41$ & $15.1 \pm 0.54$ & $14.9 \pm 0.59$ \\
\hline APTT (sec) & $13.9 \pm 0.93$ & $13.3 \pm 1.83$ & $13.6 \pm 1.10$ & $13.7 \pm 1.58$ \\
\hline Neutrophils (\%) & $12.3 \pm 3.25$ & $11.5 \pm 3.9$ & $10.8 \pm 4.2$ & $14.8 \pm 6.2$ \\
\hline Lymphocytes (\%) & $83.3 \pm 2.16$ & $84.2 \pm 3.9$ & $84.4 \pm 4.1$ & $81.2 \pm 6.5$ \\
\hline Eosinophils (\%) & $1.1 \pm 0.54$ & $1.0 \pm 0.1$ & $1.1 \pm 0.5$ & $0.9 \pm 0.3$ \\
\hline Monocytes (\%) & $1.6 \pm 0.48$ & $1.8 \pm 0.3$ & $1.8 \pm 0.4$ & $1.5 \pm 0.3$ \\
\hline Basophils (\%) & $0.5 \pm 0.15$ & $0.4 \pm 0.1$ & $0.4 \pm 0.1$ & $0.4 \pm 0.1$ \\
\hline LUC (\%) & $1.3 \pm 0.43$ & $1.1 \pm 0.4$ & $1.4 \pm 0.4$ & $1.2 \pm 0.3$ \\
\hline
\end{tabular}

Results are presented as mean $\pm \mathrm{SD}$. * Means Dunn's rank sum test significant at the 0.05 level. WBC, total leukocyte count; RBC, total red blood cell; HGB, hemoglobin; HCT, hematocrit; MCV, mean corpuscular volume; MCH, mean corpuscular hemoglobin; MCHC, mean corpuscular hemoglobin concentration; RET\%; relative reticulocyte, PLT; platelet count, PT; prothrombin time; APTT; activated partial thromboplastin time; LUC, Large unstained cells.

were not observed in a dose-dependent manner and were just observed in single-sex of rats. Therefore, we considered these findings relatively of little toxicological importance.

\section{Conclusions}

Our findings demonstrated that SHT did not have any adverse effects in both sexes of rats up to a dose of 2000 $\mathrm{mg} / \mathrm{kg} /$ day for 4 -week administration period. Minor changes were observed at $5000 \mathrm{mg} / \mathrm{kg} /$ day (the highest dose tested), but SHT did not induce gross pathological findings. Further investigation, including subchronic toxicity study, is required to establish a firm conclusion. The same dosage, as used in this study, is considered appropriate to design a subchronic toxicity study.

\section{Data Availability}

Due to organizational restrictions, the data and materials will not be available.

\section{Conflicts of Interest}

The authors declare that they have no conflicts of interest. 


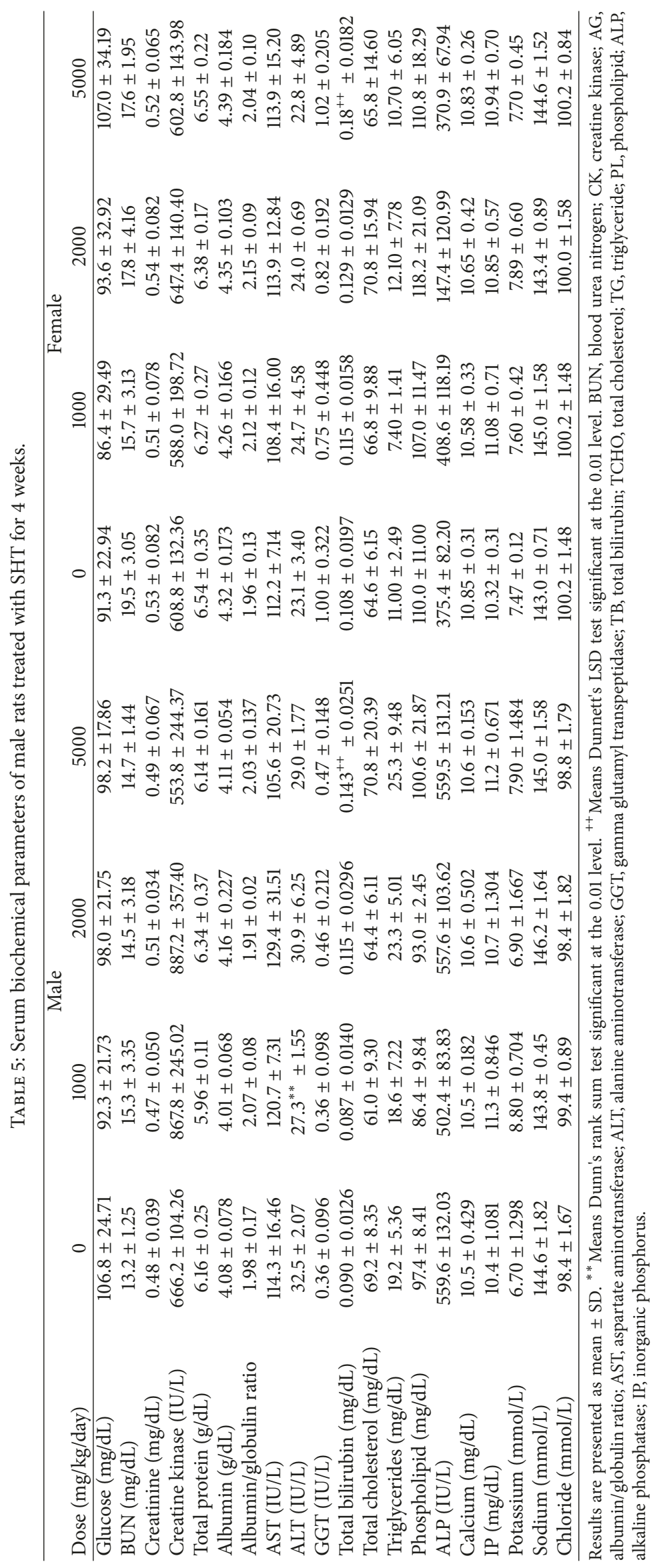


TABLE 6: Urinalysis parameters of female rats treated with SHT for 4 weeks.

\begin{tabular}{|c|c|c|c|c|}
\hline Dose (mg/kg/day) & 0 & 1000 & 2000 & 5000 \\
\hline \multicolumn{5}{|l|}{ Male } \\
\hline Volume (mL) & $21.4 \pm 5.81$ & $14.8 \pm 2.28$ & $28.4 \pm 8.65$ & $20.2 \pm 13.90$ \\
\hline Specific gravity & $1.010 \pm 0.0035$ & $1.013 \pm 0.0027$ & $1.011 \pm 0.0042$ & $1.011 \pm 0.0022$ \\
\hline $\mathrm{pH}$ & $7.0 \pm 0.00$ & $6.7 \pm 0.27$ & $7.0 \pm 0.00$ & $6.7 \pm 0.27$ \\
\hline Potassium $(\mathrm{mmol} / \mathrm{L})$ & $108.29 \pm 26.694$ & $149.69 \pm 27.031$ & $93.26 \pm 29.780$ & $95.96 \pm 28.503$ \\
\hline Chloride $(\mathrm{mmol} / \mathrm{L})$ & $41.2 \pm 9.42$ & $42.0 \pm 5.34$ & $34.2 \pm 11.30$ & $31.6 \pm 8.35$ \\
\hline Sodium (mmol/L) & $33.6 \pm 16.01$ & $38.2 \pm 7.09$ & $21.4 \pm 14.33$ & $10.6^{+} \pm 4.62$ \\
\hline Bilirubin $^{\mathrm{a}}$ & $0 / 5$ & $0 / 5$ & $1 / 5$ & $4 / 5$ \\
\hline Urobilinogen $^{\mathrm{a}}$ & $0 / 5$ & $0 / 5$ & $0 / 5$ & $0 / 5$ \\
\hline \multicolumn{5}{|l|}{ Female } \\
\hline Volume (mL) & $16.4 \pm 3.85$ & $18.6 \pm 5.08$ & $16.4 \pm 7.92$ & $10.4 \pm 4.34$ \\
\hline Specific gravity & $1.015 \pm 0.000$ & $1.013 \pm 0.0027$ & $1.015 \pm 0.0079$ & $1.017 \pm 0.0045$ \\
\hline $\mathrm{pH}$ & $6.6 \pm 0.22$ & $6.7 \pm 0.45$ & $6.3 \pm 0.84$ & $6.2 \pm 0.27$ \\
\hline Potassium $(\mathrm{mmol} / \mathrm{L})$ & $123.89 \pm 31.436$ & $101.60 \pm 34.955$ & $106.01 \pm 76.483$ & $112.67 \pm 36.457$ \\
\hline Chloride $(\mathrm{mmol} / \mathrm{L})$ & $59.0 \pm 8.43$ & $51.8 \pm 21.42$ & $57.6 \pm 43.18$ & $67.6 \pm 21.61$ \\
\hline Sodium (mmol/L) & $50.2 \pm 26.08$ & $40.0 \pm 16.69$ & $37.4 \pm 23.35$ & $37.2 \pm 13.81$ \\
\hline Bilirubin $^{\text {a }}$ & $0 / 5$ & $0 / 5$ & $1 / 5$ & $5 / 5$ \\
\hline Urobilinogen $^{\mathrm{a}}$ & $0 / 5$ & $0 / 5$ & $1 / 5$ & $3 / 5$ \\
\hline
\end{tabular}

Results are presented as mean \pm SD. ${ }^{\text {a }}$ Number of animals with sign animals/total animal number. ${ }^{+}$means Dunnett's LSD test significant at the 0.05 level.

TABLE 7: Relative organ weight (\%) of rats treated with SHT for 4 weeks.

\begin{tabular}{|c|c|c|c|c|}
\hline Dose (mg/kg/day) & 0 & 1000 & 2000 & 5000 \\
\hline \multicolumn{5}{|l|}{ Male } \\
\hline Body weight & $393.7 \pm 24.60$ & $394.2 \pm 29.80$ & $400.3 \pm 31.07$ & $393.5 \pm 30.70$ \\
\hline Brain & $0.514 \pm 0.0131$ & $0.536 \pm 0.0509$ & $0.502 \pm 0.0514$ & $0.521 \pm 0.0582$ \\
\hline Heart & $0.350 \pm 0.0390$ & $0.363 \pm 0.0150$ & $0.355 \pm 0.0478$ & $0.345 \pm 0.0312$ \\
\hline Lung & $0.398 \pm 0.0320$ & $0.384 \pm 0.0168$ & $0.394 \pm 0.0300$ & $0.395 \pm 0.0297$ \\
\hline Kidneys & $0.839 \pm 0.0346$ & $0.914 \pm 0.0379$ & $0.898 \pm 0.0603$ & $0.936 \pm 0.0673$ \\
\hline Liver & $3.295 \pm 0.1160$ & $3.331 \pm 0.2191$ & $3.427 \pm 0.2521$ & $3.740^{++} \pm 0.1483$ \\
\hline spleen & $0.192 \pm 0.0371$ & $0.207 \pm 0.0251$ & $0.189 \pm 0.0277$ & $0.238 \pm 0.0566$ \\
\hline Testes & $0.860 \pm 0.0642$ & $0.798 \pm 0.0494$ & $0.823 \pm 0.0751$ & $0.860 \pm 0.0670$ \\
\hline Prostate & $0.135 \pm 0.0316$ & $0.123 \pm 0.0290$ & $0.100 \pm 0.0235$ & $0.122 \pm 0.0142$ \\
\hline Epididymis & $0.273 \pm 0.0298$ & $0.263 \pm 0.0201$ & $0.260 \pm 0.0284$ & $0.304 \pm 0.0584$ \\
\hline Thymus & $0.127 \pm 0.0132$ & $0.114 \pm 0.0215$ & $0.158 \pm 0.0316$ & $0.147 \pm 0.0152$ \\
\hline THPA & $0.005 \pm 0.0006$ & $0.006 \pm 0.0010$ & $0.006 \pm 0.0010$ & $0.006 \pm 0.0015$ \\
\hline PITG & $0.003 \pm 0.0002$ & $0.003 \pm 0.0002$ & $0.003 \pm 0.0003$ & $0.003 \pm 0.0002$ \\
\hline ADRG & $0.016 \pm 0.0029$ & $0.017 \pm 0.0032$ & $0.015 \pm 0.0027$ & $0.014 \pm 0.0006$ \\
\hline SVCG & $0.347 \pm 0.0472$ & $0.369 \pm 0.0542$ & $0.338 \pm 0.0415$ & $0.365 \pm 0.0836$ \\
\hline SALG & $0.182 \pm 0.0297$ & $0.191 \pm 0.0137$ & $0.179 \pm 0.0115$ & $0.184 \pm 0.0226$ \\
\hline \multicolumn{5}{|l|}{ Female } \\
\hline Body weight & $227.4 \pm 14.60$ & $227.1 \pm 18.89$ & $230.6 \pm 17.99$ & $217.6 \pm 18.29$ \\
\hline Brain & $0.809 \pm 0.0497$ & $0.822 \pm 0.0858$ & $0.844 \pm 0.0774$ & $0.854 \pm 0.0482$ \\
\hline Heart & $0.376 \pm 0.0236$ & $0.399 \pm 0.0269$ & $0.375 \pm 0.0166$ & $0.394 \pm 0.0179$ \\
\hline Lung & $0.524 \pm 0.0063$ & $0.520 \pm 0.0204$ & $0.489 \pm 0.0320$ & $0.515 \pm 0.0258$ \\
\hline Kidneys & $0.899 \pm 0.0173$ & $0.953 \pm 0.0684$ & $0.943 \pm 0.0432$ & $1.016^{+} \pm 0.0595$ \\
\hline Liver & $3.534 \pm 0.0908$ & $3.584 \pm 0.1903$ & $3.698 \pm 0.1469$ & $3.914^{+} \pm 0.1393$ \\
\hline Spleen & $0.236 \pm 0.0322$ & $0.244 \pm 0.0228$ & $0.237 \pm 0.0211$ & $0.299^{+} \pm 0.0198$ \\
\hline Ovaries & $0.047 \pm 0.0033$ & $0.043 \pm 0.0065$ & $0.415 \pm 0.0059$ & $0.045 \pm 0.0072$ \\
\hline UTEC & $0.255 \pm 0.1209$ & $0.210 \pm 0.0159$ & $0.243 \pm 0.0596$ & $0.248 \pm 0.0500$ \\
\hline Thymus & $0.177 \pm 0.0225$ & $0.196 \pm 0.0078$ & $0.209 \pm 0.0347$ & $0.191 \pm 0.0247$ \\
\hline THPA & $0.008 \pm 0.0015$ & $0.008 \pm 0.0007$ & $0.008 \pm 0.0016$ & $0.009 \pm 0.0013$ \\
\hline PITG & $0.006 \pm 0.0006$ & $0.006 \pm 0.0004$ & $0.006 \pm 0.0007$ & $0.007 \pm 0.0005$ \\
\hline ADRG & $0.034 \pm 0.0054$ & $0.031 \pm 0.0034$ & $0.033 \pm 0.0065$ & $0.035 \pm 0.0049$ \\
\hline SALG & $0.206 \pm 0.0156$ & $0.200 \pm 0.0149$ & $0.194 \pm 0.0121$ & $0.202 \pm 0.0225$ \\
\hline
\end{tabular}

Results are presented as mean \pm SD. ${ }^{+}$Means Dunnett's LSD test significant at the 0.05 level. ${ }^{++}$Means Dunnett's LSD test significant at the 0.01 level. THPA, thyroid and parathyroid glands; PITG, pituitary gland; ADRG, adrenal glands; SVCG, seminal vesicles with coagulating gland; SALG, salivary glands; UTEC, Uterus/cervix. 


\section{Authors' Contributions}

Mee-Young Lee and Hyekyung Ha conceived and designed the study. Su-Cheol Han performed the experiments. ChangSeob Seo extracted herbal formula and performed HPLC analysis of SHT decoction. Sae-Rom Yoo analyzed the data. Chang-Seob Seo and Sae-Rom Yoo wrote a draft manuscript. Sae-Rom Yoo, Chang-Seob Seo, Mee-Young Lee, Hyekyung $\mathrm{Ha}$, and Hyeun-kyoo Shin edited and revised manuscript. All authors read and approved the final manuscript.

\section{Acknowledgments}

This research was supported through the "Construction of Scientific Evidences for Herbal Medicine Formulas (K17251 and KSN1812240)" grant from the Korea Institute of Oriental Medicine.

\section{References}

[1] J. Heo, Donguibogam, Ministry of Health \& Welfare, Seoul, Korea, 2013.

[2] J.-W. Ko, N.-R. Shin, S.-H. Park et al., "Ssanghwa-Tang, a traditional herbal formula, suppresses cigarette smoke-induced airway inflammation via inhibition of MMP-9 and Erk signaling," Molecular \& Cellular Toxicology, vol. 13, no. 3, pp. 295-304, 2017.

[3] K.-S. Shim, J.-H. Lee, C. J. Ma et al., "Inhibitory effect of Ssanghwa-tang on bone loss in ovariectomized rats," Animal Cells and Systems, vol. 14, no. 4, pp. 283-289, 2010.

[4] A. Kim, N.-H. Yim, M. Im et al., "Ssanghwa-tang, an oriental herbal cocktail, exerts anti-melanogenic activity by suppression of the p38 MAPK and PKA signaling pathways in B16F10 cells," BMC Complementary and Alternative Medicine, vol. 13, no. 1, p. 214, 2013.

[5] OECD, Acute Oral toxicity - Acute Toxic Class Method, 2001, https://read.oecd-ilibrary.org/environment/test-no-423acute-oral-toxicity-acute-toxic-class-method_978926-4071001en\#page4.

[6] S. J. Kim, M. Y. Lee, I. S. Shin et al., "Single dose aute toxicity of Ssanghwa-tang in Crl:CD (SD) rats," The Korea Association of Herbology, vol. 26, no. 2, pp. 39-43, 2011.

[7] S. Parasuraman, "Toxicological screening," Journal of Pharmacology and Pharmacotherapeutics, vol. 2, no. 2, pp. 74-79, 2011.

[8] C. S. Seo, J. H. Kim, and H. K. Shin, "High performance liquid chromatographic simultaneous analysis for quantitation of eight marker compounds of traditional Korean Medicine, Ssanghwa-Tang," Asian Journal of Chemistry, vol. 26, no. 10, pp. 2877-2882, 2014.

[9] J. Y. Ma, D. H. Park, K. S. Park, K. Y. Do, and H. K. Shin, "Acute toxicity study on ssanghwatang in mice," The Korean Jounal of Oriental Medicine, vol. 13, no. 1, pp. 161-164, 2007.

[10] S. R. Yoo, H. Ha, M. Y. Lee et al., "A 4-week repeated-dose oral toxicity study of bojungikgi-tang in Crl:CD Sprague dawley rats," Evidence-Based Complementary and Alternative Medicine, vol. 2017, Article ID 4748904, 8 pages, 2017.

[11] E. Piva, C. Brugnara, F. Spolaore, and M. Plebani, "Clinical utility of reticulocyte parameters," Clinics in Laboratory Medicine, vol. 35, no. 1, pp. 133-163, 2015.

[12] M. Giknis and Clifford, Clinical Laboratory Parameters for Crl:CD (SD) Rats, 2006. 


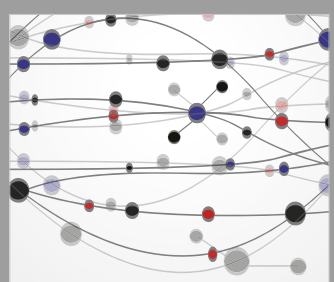

The Scientific World Journal
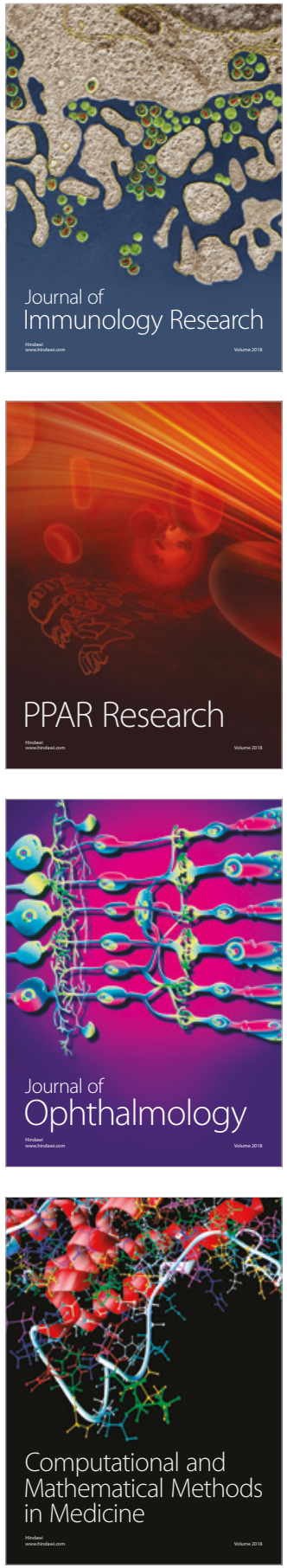

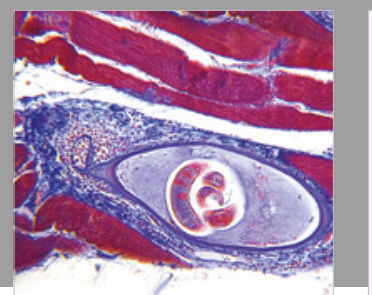

Gastroenterology Research and Practice

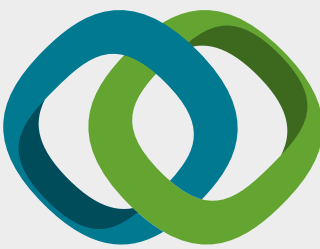

\section{Hindawi}

Submit your manuscripts at

www.hindawi.com
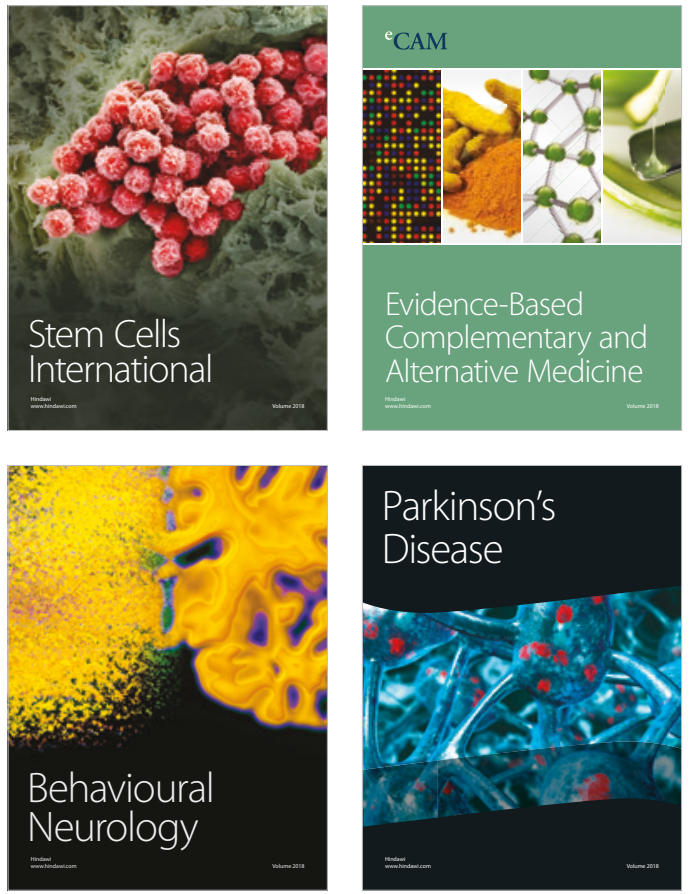

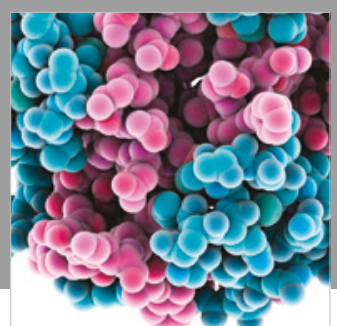

ournal of

Diabetes Research

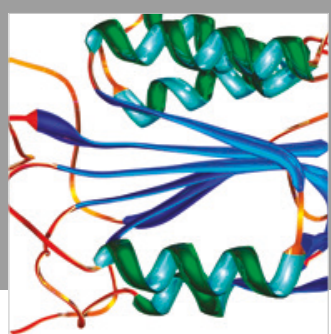

Disease Markers
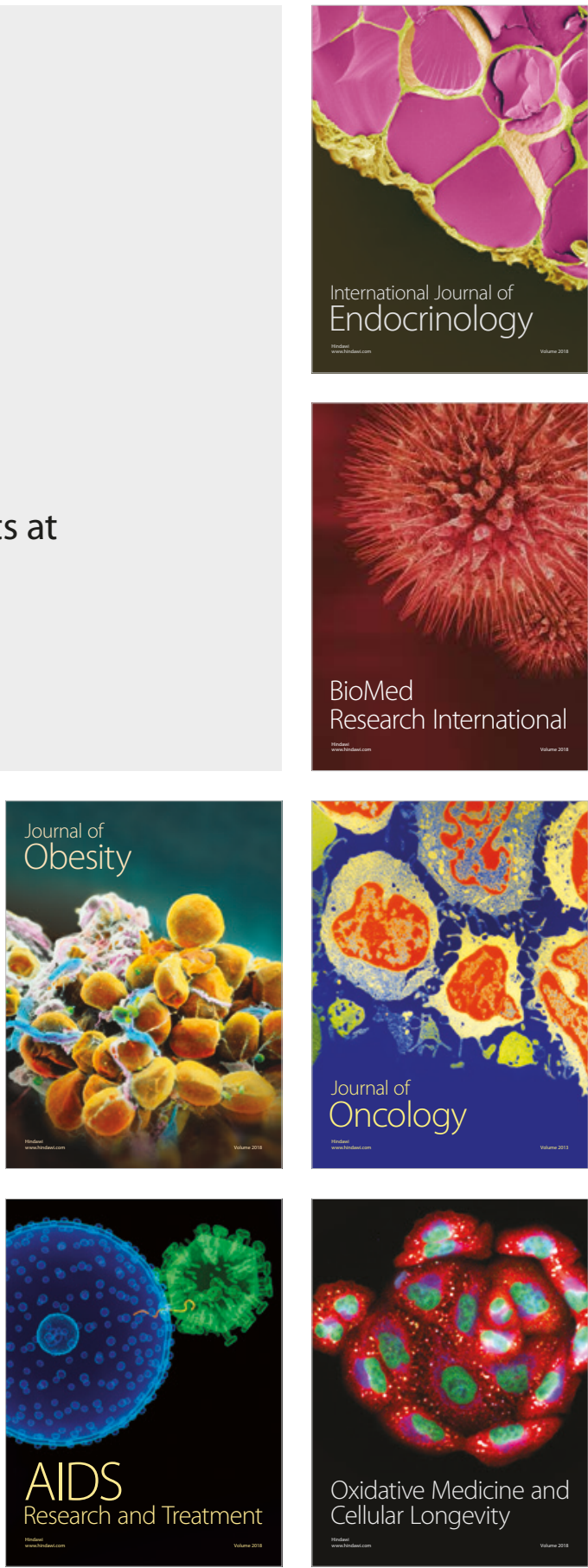